\title{
ENTREVista CON Virginia BERTOLOTTI
}

\author{
Leandra Cristina de Oliveira | Lattes | leandraletras@hotmail.com \\ Universidade Federal de Santa Catarina
}

Izete Lehmkuhl Coelho | Lattes | izete.lehmkuhl.coelho@ufsc.br Universidade Federal de Santa Catarina | CNPq

\section{Introducción}

Para representar el mundo hispánico, invitamos a la académica uruguaya Virginia Bertolotti, cuyas contribuciones en el ámbito de las investigaciones sobre las formas de tratamiento en el español hispanoamericano son de gran importancia y con quien hemos cruzado camino en los eventos y proyectos sobre el tema al que se orienta esta publicación.

Bertolotti es Doctora en Humanidades y Artes, Profesora Titular del Departamento de Medios y Lenguajes de la Facultad de Información y Comunicación de la Universidad de la República, investigadora de Nivel II del Sistema Nacional de Investigadores y Académica de Número de la Academia Nacional de Letras del Uruguay.

La Dra. Bertolotti ha centrado sus intereses académicos en la conformación histórica del español en América, en la gramática y en la enseñanza de la lengua escrita. Dirigió el Programa de Lectura y Escritura en español (Administración Nacional de Educación Pública, Uruguay) y codirige con Concepción Company Company el Corpus Diacrónico y Diatópico del Español de América (CORDIAM). Es autora de $\ll A$ mí de vos no me trata ni usted ni nadie». Sistemas e historia de las formas de tratamiento en el español en América, coautora con Magdalena Coll de tres obras: Retrato lingüistico del Uruguay, de Documentos para la historia del español en el Uruguay, en la que también participa Ana Polakof, y de Documentos para la historia del portugués en el Uruguay, en colaboración con Serrana Caviglia y Marianela Fernández. Además, ha compilado recientemente un volumen titulado Comunicación, lengua e información y ha publicado numerosos artículos en libros y revistas de América, Estados Unidos y Europa.

En esta entrevista, la lingüista nos expone brevemente los caminos recorridos tras la publicación de su tesis en el año de 2011, los retos, las aspiraciones y las grietas en el campo de la investigación sobre las formas de tratamiento. 
Además de brindarnos informaciones sobre el proceso de escritura y publicación de su reconocida obra «A mí de vos no me trata ni usted ni nadie». Sistema e historia de las formas de tratamiento en la lengua española en América (BERTOLOTTI, 2015), Bertolotti menciona las contribuciones que han aportado los estudios publicados por los brasileños Célia Lopes, Leonardo Marcotulio y sus equipos, y por el romanista Martin Hummel, sin con esto dejar de recordar la importancia de trabajos del siglo xx, como el de la hispanista argentina Frida Weber de Kurlat de la década de 1940, el trabajo de José Pedro Rona de $1967^{1}$ y las diversas publicaciones de María Beatriz Fontanella de Weinberg sobre el tema de las formas de tratamiento.

El rescate de la historia y de los pasos perseguidos por ella misma y por otros investigadores acerca del fenómeno se desarrolla de forma relativamente sencilla en esta entrevista, la cual, a pesar de no haber tenido las pretensiones de un debate científico, llega a serlo con el tono de una exposición académica, pero informal y diversa.

\section{Entrevista}

Oliveira y Coelho: Conviene empezar esta entrevista destacando las contribuciones que ha aportado su tesis doctoral intitulada Los cambios en la segunda persona del singular durante el siglo XIX en el español del Uruguay (BERTOLOTTI, 2011) al campo de las investigaciones de las formas de tratamiento en el español hispanoamericano. Desde ahí, creemos interesante empezar por las siguientes cuestiones: en la actualidad ;por cuáles desdoblamientos han pasado sus investigaciones sobre el fenómeno? ¿Con qué retos se ha enfrentado?

Virginia Bertolotti: Mi mayor reto en este momento es conseguir abandonar el tema de las formas de tratamiento. Desde hace muchos años vengo indagando sobre esto y me gustaría ya dejarlo y dedicarme a otros temas con la misma intensidad. Sin embargo, me siguen apareciendo cuestiones sin resolver, nuevas curiosidades y por lo tanto, sigo investigando.

Por ejemplo, en este momento, luego de haber logrado comprender, creo, por qué la forma vosotros no está presente en el español americano, que era un tema que tenía pendiente y me molestaba mucho no entender cabalmente, estoy tratando ahora — y cuando digo ahora, es al mismo tiempo que estoy respondiendo a estas preguntas — de entender exactamente cuáles fueron las razones por las cuales, cuando la forma ya estaba en desuso

\footnotetext{
${ }^{1}$ La entrevistada se refiere a la publicación RONA, J. P. Geografía y morfología del voseo. Porto Alegre: Pontificia Universidad Católica, 1967.
} 
en América, reaparece con mucha presencia — parece revivir - en la prensa americana, entonces emergente. $\mathrm{Y}$ ahora me está surgiendo un problemita nuevo consistente en la relación irregular entre los singulares y sus aparentemente respectivos plurales.

En cuanto a mi tesis, que tiene ya más de diez años, creo que el aporte fundamental está en la explicación histórica de la actual extensión del voseo en América Latina, explicación que surge de la relectura de la bibliografía y del empleo de fuentes complementarias (fuentes literarias, comentarios metalingüísticos).

Oliveira y Coelho: $i S u$ trayectoria teórico-metodológica ha cambiado en los últimos años? Si es el caso, ¿a qué se lo atribuye?

Virginia Bertolotti: No sé si mi trayectoria ha cambiado. Es más, no sé si tengo una trayectoria. Pero si miro hacia atrás, lo que veo es una preocupación cada vez mayor por la calidad de los datos con los cuales hacemos historia de la lengua y por la forma de interpretarlos.

¿Qué quiero decir con la forma de interpretarlos? Creo que dos cosas. Por un lado, que a diferencia de lo que sucede con la lingüística brasileña, la lingüística hispánica es muy europeísta. Quizá es un poco largo para explicar aquí, pero quienes nos ocupamos del español en las Américas (seamos latinoamericanos, alemanes, europeos o estadounidenses) solemos sesgar la mirada de los datos focalizando en el resultado europeo (o castellano) de los procesos de cambio lingüístico. De alguna manera, no solo los hablantes americanos tienen una representación europeísta de la lengua española, sino que también la tenemos los lingüistas. Por otro, y también, a diferencia de lo que sucede en buena parte de la lingüística histórica brasileña, el peso que se le da al contacto lingüístico con hablantes no europeos al hacer historia del español de América es muy bajo. Suele pensarse siempre en españoles (europeos) hablando entre sí. En el caso del Uruguay, mi país, se estudia también el contacto con el portugués, pero no se suele pensar en un contexto comunicativo plurilingüe que, a mi juicio, permite comprender mejor algunos fenómenos lingüísticos clásicos en los estudios hispánicos, como es el de las formas de tratamiento.

Oliveira y Coelho: Hablemos un poco sobre su libro «A mí de vos no me trata ni usted ni nadie». Sistema e historia de las formas de tratamiento en la lengua española en América (BERTOLOTTI, 2015), que ocupó el primer lugar en los Premios Anuales de Literatura 2014 por el Ministerio de Educación y Cultura de Uruguay. La obra se introduce de for- 
ma muy didáctica familiarizando al lector inexperto sobre la temática del tratamiento. En las dos partes siguientes presenta, respectivamente, una revisión crítica y profunda de la historia de los sistemas de tratamiento en el español preamericano y americano y la síntesis de su tesis doctoral sobre la diacronía de los verbos y pronombres alocutivos singulares en el español en Uruguay. ¿Cómo fue su experiencia al escribir esta obra de gran relevancia para hispanistas y romanistas?

Virginia Bertolotti: Cansadora [risas]. Supongo que como le sucede a todos quienes investigan y escriben sobre sus investigaciones, el proceso de escritura, sobre todo en las últimas etapas, es tedioso, fastidioso y nos obliga a poner en juego habilidades que nos cuesta ligar con los procesos investigativos. Cuando sentimos que ya terminamos, hay que empezar de nuevo. Yo tuve la enorme suerte de que los dos revisores que evaluaron la obra hicieron una lectura muy fina y muy constructiva y por lo tanto trabajar con sus observaciones me ayudó mucho a mejorar el texto. La correctora de la UNAM, María del Refugio Campos Guardado, que trabajó con mi libro, es alguien de una calidad y profesionalidad enormes y eso también ayudó mucho a que la versión final fuera mucho mejor que si hubiera quedado solo en mis manos. $\mathrm{Y}$ en plan de agradecer, también me cabe agradecer a una estudiante de un curso que di en México que fue quien me dio la idea de escribir ese libro. Me preguntó si lo que estaba diciendo estaba escrito en algún lado, y Concepción Company Company, que estaba presente en ese momento, recogió el guante por mí y me impulsó a escribirlo.

Oliveira y Coelho: En las últimas décadas, las investigaciones sobre las formas y fórmulas de tratamiento se han ampliado y diversificado en términos teórico-metodológicos. Podemos considerar, a título de ejemplos, el libro organizado por Martin Hummel, Bettina Kluge y María Eugenia Vázquez Laslop Formas y fórmulas de tratamiento en el mundo hispánico (2010), que reúne 46 capítulos sobre el tema, el que organizó Leticia Rebollo Couto y Célia Regina dos Santos Lopes (2011) As formas de tratamento em português e em espanhol: variação, mudança e funções conversacionais, las secciones temáticas en eventos de gran relevancia, como los congresos internacionales de la Asociación de Lingüística y Filología de América Latina, los eventos específicos sobre el tema, como las tres ediciones del Congreso «Formas y fórmulas de tratamiento del mundo hispánico, luso y brasileño» y la revista que aquí se presenta. ¿A qué se debe la gran atención dedicada a ese fenómeno lingüístico? 
Virginia Bertolotti: Efectivamente, en el siglo XXI pareciera que ha habido una explosión en los estudios sobre tratamiento. No debemos olvidar otro antecedente como fue el Coloquio de París, del Foro de Lenguas Europeas de 2003, que estuvo dedicado a los pronombres de segunda persona y formas de tratamiento en las lenguas de Europa. Los hispanistas debemos agradecer a Martin Hummel la convocatoria al congreso que dio origen al libro del 2010, que es un texto fundamental. No solamente por la cantidad y calidad de los trabajos que allí se encuentran, sino porque ese libro nos dio realmente una mirada panorámica de qué se sabía y de cómo se venían estudiando los tratamientos (y también de los baches de conocimiento, por supuesto).

Me da la impresión de que el interés se debe a que es un tema muy interesante, pero supongo que todos los temas lo son [risas]. Hablando en serio, el español es una de las lenguas con más extensión geográfica en el mundo y por su complejo proceso de difusión y por los variadísimos entramados socioculturales que la sustentan (¿o que ella sustenta?) la variación en los tratamientos es mucha y en muchos casos asociada a las identidades nacionales o locales. Creo que por eso interpela a los estudiosos, también a los hablantes y muchas veces interpela también a quienes toman decisiones para los sistemas educativos y para la financiación de la investigación.

Por otro lado, la proliferación de estudios también puede explicarse porque es un objeto que en apariencia no requiere de muchos conocimientos previos o es de fácil acceso para los principiantes. No es lo mismo estudiar la variación gramatical que la variación léxica y este tema en alguna medida se parece al léxico, sin tener las dificultades del estudio del léxico ya que los tratamientos gramaticales constituyen sistemas o microsistemas. Además, aunque parece algo muy sencillo, es en realidad muy complejo y por lo tanto permite varios enfoques. Esas son las razones, además de la proliferación general de estudios existentes sobre casi todos los temas, que se dan por las facilidades en el acceso a los datos y a la bibliografía de los últimos años. Y también por los cambios en la economía de la academia, que presiona para la producción (creando este sobrecalentamiento académico en el que estamos, en que se publica más de lo que podemos leer), pero ya nos derivaríamos a hablar de otras cuestiones.

Oliveira y Coelho: Entre las investigaciones sobre los sistemas de tratamiento de segunda persona del mundo hispanoamericano, ¿̇cuáles aportes merecen destacarse y cuáles son los retos por enfrentar? 
Virginia Bertolotti: Más que de lo actual, prefiero hablar de trabajos del siglo xx, que a veces, en la presión de la lectura de los últimos trabajos olvidamos que existen. Entre ellos me interesa recordar el fino trabajo de la hispanista argentina Frida Weber de Kurlat de la década de 1940, el trabajo de Rona de 1967 y los varios trabajos sobre la temática de María Beatriz Fontanella de Weinberg.

Entiendo que tenemos a estas alturas una acumulación suficiente de descripciones de sincronías, y si bien podrían seguirse haciendo estudios puntuales, me parece que ya estamos en condiciones de extraer algunas regularidades interesantes. Por un lado, porque sería muy bueno ir tras los procesos globales que permitan el trazado o los trazados de la lengua española y poder salir de la mirada anecdótica. Y por otro, porque es seguro que iluminarían sobre las formas de tratamientos en particular, pero también en general sobre las lenguas naturales.

Esa acumulación, en parte sistematizada por Moyna hace unos años y por mí hace un poco menos de tiempo, ha sido todavía poco puesta en diálogo con lo que se sabe del portugués de Brasil, en donde también se ha avanzado muchísimo gracias a los trabajos de Leonardo Marcotullio y de Célia Lopes y sus equipos. Este sería un paso para hacer aportes desde la lingüística romance a la lingüística general.

En la diacronía de los tratamientos resta mucho camino por recorrer, ya que hay zonas de las Américas de las que poco sabemos todavía, y, lamentablemente, no solo en el campo de los estudios históricos de los tratamientos. Entre lo poco estudiado aún en el campo de las formas de tratamientos están los honoríficos, que, salvo su merced (y vuestra merced) casi no han sido investigados en el español hispanoamericano en perspectiva histórica.

Y hay por lo menos tres pendientes más. Hacer una buena síntesis del voseo, la estrella de los tratamientos americanos. Yo tengo el título de ese trabajo, pero no el tiempo para hacerlo: debiera llamarse Qué hay de nuevo sobre el viejo «vos». También es necesario hacer un buen estudio gramatical histórico y sincrónico comprensivo de esta forma. Por último, y como ya ha dicho muchas veces Mayte García Godoy, es imperioso que hagamos estudios más sintagmáticos, considerando sistemáticamente todas las codificaciones del tratamiento, las pronominales, la verbales y las nominales y no focalizando solo en alguna de ellas alternativamente.

Oliveira y Coelho: Últimamente, a partir de las consideraciones de Eckert (2005, 2008, 2012, 2018) sobre las tres olas por las que pasa la Sociolingüística, fenómenos lingüísticos diversos han sido estudiados a la luz de un redireccionamiento en la interpretación 
del significado de la variación, considerando, más allá del vínculo con las variables macrosociológicas, las variables microsociológicas. ¿En qué medida el marco de la tercera ola de la Sociolingüística, más centrada en el significado como indexador de identidad y, por tanto, como práctica social, indicadora y reproductora, puede contribuir para la descripción del fenómeno de las formas y fórmulas de tratamiento? ¿En qué medida es posible tomar provecho de las tres perspectivas/olas de la Sociolingüística ampliamente debatida en las publicaciones de Penelope Eckert?

Virginia Bertolotti: Insertos o no en la Tercera ola, muchos de quienes estudiamos formas de tratamiento hemos apelado a la indexación social para comprenderlas mejor. De hecho, en este trabajo que mencioné más arriba, lo que estoy encontrando es que ese uso de vosotros de la prensa en el siglo xIx se empleaba como una forma de indexación de grupos cuyos rasgos constituidos o imaginarios eran posturas políticas, sociales e ideológicas. En este sentido los vosotros eran "amantes de la libertad" o eran "buenos americanos", pero no eran vosotros los "señores editores" o los "queridos amigos". Efectivamente, las viejas y fructíferas categorías sociales del tratamiento como el poder, la solidaridad, la clase social, la distancia por clase o por sexo no permiten dar cuenta de algunos de los comportamientos lingüísticos de los hablantes. Necesitamos otras. De hecho, cuando queremos dar cuenta de una realidad muy compleja como la de los sistemas de tratamiento hispánicos, inevitablemente recurrimos a ellas, a las macrocategorías, para dar una descripción comprensible y abarcativa, aun a sabiendas de que estamos simplificando. Pero cuando queremos comprender el entramado fino o cuando queremos además de describir, explicar, esto es, comprender el porqué del uso o el cómo y porqué del cambio lingüístico, aquellas categorías macro, propias de la primera sociolingüística, a veces nos resultan escasas. Y entiendo que se pueden superar tanto desde la reintroducción de la estilística que supone la tercera ola o a través de los estudios de la variación textual de la romanística alemana y coseriana.

Oliveira y Coelho: Los estudios de las formas y fórmulas de tratamiento, más allá de los intereses de la Sociolingüística, se han fundamentado en distintas bases teóricas, como la Lingüística Histórica, la Pragmática y la Socio-pragmática. ¿A qué se atribuye ese apego a tan distintos campos teóricos de la Lingüística?

Virginia Bertolotti: A que son en apariencia simples pero en realidad muy complejas y, por tanto, enfocables desde diversos subcampos de la lingüística. Tienen un componente 
gramatical, pero, a diferencia de otras zonas de la gramática, las formas y fórmulas de tratamiento cambian con cierta rapidez y evidencia, y eso las hace atractivas para el estudio histórico.

Oliveira y Coelho: Podría usted comentar sobre la posibilidad y la conveniencia de superar los límites de la Lingüística y expandir la reflexión sobre la variación y el cambio en el sistema de las formas de tratamiento de las diferentes lenguas a otras áreas del conocimiento y a otros campos de actuación.

Virginia Bertolotti: Siempre es posible y conveniente superar los límites de los campos del conocimiento. De hecho, la Lingüística como campo en su escaso siglo de vida ha revisado y ampliado sus límites. Nunca había pensado en esta pregunta, y mi tentación inicial es decir que el conocimiento de los estudios sobre tratamiento sería de pertinencia para otros campos de las ciencias sociales y humanas incluyendo en esta la geografía, los estudios sobre migraciones, sin duda, pero también para la minería de datos, para el procesamiento de Big Data proveniente de la comunicación interpersonal mediada por tecnologías, por decir algo.

Oliveira: Como hablante no nativa del español e investigadora interesada en los polifacéticos pronombres de tratamiento y en su multifuncionalidad en las interacciones entre hispanohablantes de los más distintos rincones, tengo que confesarle que no me resulta fácil elegir el tratamiento oportuno en la presente interlocución. Si bien existe la formalidad requerida por el género textual, nos encontramos en cierta simetría social aparte de que usted es uruguaya (y por ello aquí casi me sale un "sos"). ¿Qué podría decirme sobre este drama en que comúnmente se encuentra un hablante no nativo del español? ¿Se me permitiría usar el trato vos o "a usted de vos no le trata" un extranjero?

Virginia Bertolotti: Me gusta mucho la exageración de llamarlo "drama”, porque efectivamente, al hablar español, en muchos casos, estamos frente a pequeños dramas al decidir qué forma de tratamiento usar. Sería muy adecuado para mí que nos tratáramos con una forma solidaria ya que somos colegas y tenemos menos de una generación de diferencia de edad. Sos más joven que yo pero no podrías ser mi hija, me parece. Sin embargo, como estamos en una interacción algo formal (una entrevista) y además pública, también estaría bien un tratamiento de usted, aunque fuera algo anticuado en el contexto rioplatense. La retracción de usted es creciente en el mundo hispánico, pero es muy irregular cuánto 
se ha retraído en el correr del último siglo. En México creo que solo habría tratamiento de usted en esta entrevista. A mí me resulta muy raro que me trates de usted. Entonces, nos quedan dos posibilidades tú o vos. Siendo extranjera, si me trataras de vos supondría que aprendiste español en inmersión en el Río de la Plata o en alguna zona voseante. Los uruguayos que tenemos tres formas para el singular, tú, vos y usted, esperamos y solemos dar a los extranjeros tú, porque el tratamiento voseante está en nuestra representación lingüística de lo rioplatense. Como siempre, esto se da en las formas más visibles, que son las pronominales; en los verbos mayoritariamente voseamos, aun sin quererlo.

Oliveira: Muito obrigada!

Virginia Bertolotti: Gracias a ti, bueno, a ustedes.

\section{Referências}

BERTOLOTTI, Virginia. A mí de vos no me trata ni usted ni nadie. Sistemas e historia de las formas de tratamiento en la lengua española en América. Ciudad de México: Universidad Nacional Autónoma de México y Universidad de la República de Uruguay, 2015.

BERTOLOTTI, Virginia. Los cambios en la segunda persona del singular durante el siglo XIX en el español del Uruguay Tese (Doutorado). Universidad Nacional de Rosario, 2011.

COUTO, Leticia Rebollo; LOPES; Célia Regina dos Santos (Org). As formas de tratamento em português e em espanhol: variação, mudança e funções conversacionais. Rio de Janeiro: Ed. da Univ. Federal Fluminense, 2011.

ECKERT, Penelope; WENGER, Etienne. Communities of practice in sociolinguistics: What is the role of power in sociolinguistic variation? Journal of Sociolinguistics, v. 9, n. 4, p. 582-589, 2005.

ECKERT, Penelope. Meaning and linguistic variation: The third wave in sociolinguistics. Cambridge: Cambridge University Press, 2018.

ECKERT, Penelope. Three waves of variation study: The emergence of meaning in the study of sociolinguistic variation. Annual review of Anthropology, v. 41, p. 87-100, 2012.

ECKERT, Penelope. Variation and the indexical field 1. Journal of sociolinguistics, v. 12, n. 4, p. 453-476, 2008.

HUMMEL, Martin; KLUGE, Bettina; LASLOP, María Eugenia Vázquez (Org.). Formas y fórmulas de tratamiento en el mundo hispánico. Ciudad de México: El Colegio de México / Centro de Estudios Lingüísticos y Literarios, 2010.

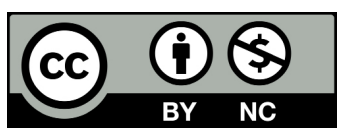

